\title{
Ultrasound Detection of Lacunae-like Image of a Cesarean Scar Pregnancy in the First Trimester
}

\author{
Atsuko Sekiguchi ${ }^{1}$, Naotaka Okuda ${ }^{1}$, Ikuno Kawabata ${ }^{1}$, \\ Akihito Nakai ${ }^{1}$ and Toshiyuki Takeshita ${ }^{2}$ \\ ${ }^{1}$ Department of Obstetrics and Gynecology, Nippon Medical School Tama Nagayama Hospital \\ ${ }^{2}$ Department of Obstetrics and Gynecology, Nippon Medical School Hospital
}

\begin{abstract}
Cesarean scar pregnancy is one of the rare types of ectopic pregnancy. Ultrasonography enables early diagnosis and the successful preservation of the uterus. However, the correct diagnosis of cesarean scar pregnancy can be difficult in some cases. We describe a case of cesarean scar pregnancy that was initially misdiagnosed on the basis of current ultrasonographic criteria. Ultrasonographic images at 9 weeks' gestation demonstrated no gestational sac but did show a bulging mass in uterine wall with irregularly shaped hypoechoic areas, which resembled the lacunae in placenta previa. Color Doppler imaging demonstrated that the lacunae-like areas were richly perfused. Cesarean scar pregnancy was finally diagnosed with magnetic resonance imaging, which showed a lack of myometrium in the lower anterior uterine wall with placental invasion. Histological examination of the uterus after hysterectomy revealed the direct invasion of trophoblasts into the mural zone which had resulted in a deficit of myometrium at the previous cesarean scar. A mass in the myometrium with richly perfused lacunae-like areas should be considered as one of the important ultrasonographic findings indicating cesarean scar pregnancy.
\end{abstract}

(J Nippon Med Sch 2013; 80: 70-73)

Key words: cesarean scar pregnancy, ultrasound, lacunae, placenta accreta

\section{Introduction}

Cesarean scar pregnancy is one of the rare types of ectopic pregnancy. The ratio of cesarean scar pregnancies to normal pregnancies has been reported to range from $1: 1,800$ to $1: 2,216^{1}$. Ultrasonography enables early diagnosis and preservation of the uterus ${ }^{2-4}$. However, differentiating cesarean scar pregnancy from abortion and cervicoisthmic pregnancy can be difficult, and misdiagnosis cannnot be completely avoided $^{5.6}$. We describe a case of cesarean scar pregnancy with atypical ultrasonographic images which could not be diagnosed with current criteria.

\section{Case Report}

A 36-year-old woman, gravida 2 para 2, visited our hospital for evaluation of amenorrhea. The obstetric

Correspondence to Atsuko Sekiguchi, Department of Obstetrics and Gynecology, Nippon Medical School Tama Nagayama Hospital, 1-7-1 Nagayama, Tama, Tokyo 206-8512, Japan

E-mail: oya-a@nms.ac.jp

Journal Website (http://www.nms.ac.jp/jnms/) 
history included 2 low-transverse cesarean sections at term. Transvaginal ultrasonography showed an echo-free space with a rim in the uterine cavity, which was diagnosed as a gestational sac at 5 weeks' gestation (Fig. 1). At 8 weeks' gestation, a fetal heart beat was not detected, and a missed abortion was diagnosed. On admission for dilatation and curettage at 9 weeks' gestation, the echo-free area in the uterine cavity was recognized as an empty sac because a trophoblastic rim appeared to be present.

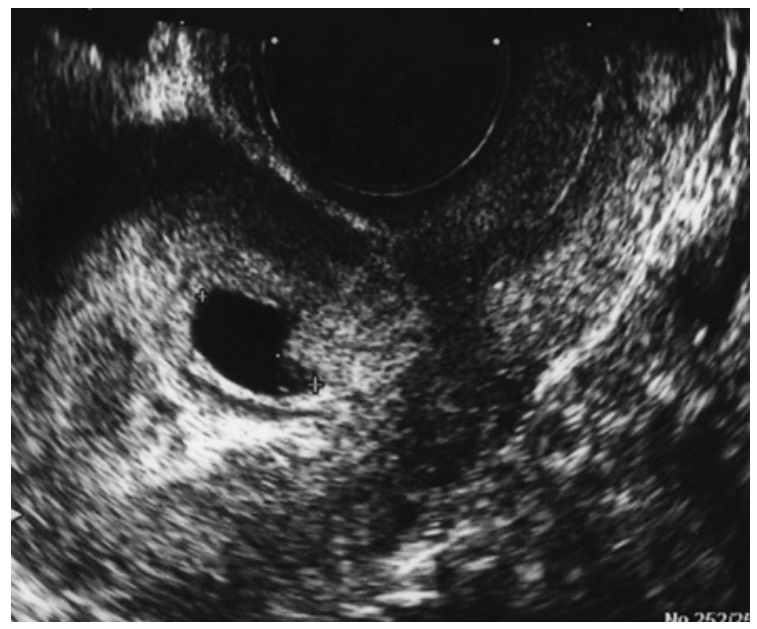

Fig. 1 Transvaginal ultrasonographic image at 5 weeks' gestation. An echo-free area with a diameter of $1.48 \mathrm{~cm}$ in the uterine cavity was recognized as a gestational sac.
Simultaneously, a bulging mass in the lower segment of the anterior uterine wall was noticed. The mass included multiple irregularly shaped hypoechoic areas, strikingly similar to lacunae (Fig. 2a). Before curettage, a 3-mm dilator was inserted into the cervix. When the dilator was removed 2 hours later, an acute hemorrhage of approximately $200 \mathrm{~mL}$ developed. The dilatation and curettage was immediately discontinued; the hemorrhage was controlled with intravaginal tampon packing.

On the same day, color Doppler imaging demonstrated that the lacunae-like areas were richly perfused (Fig. 2b). Magnetic resonance imaging showed a lack of myometrium in the lower anterior uterine wall with conceptus invasion, and the presumed sac was determined to be a fluid collection (Fig. 3). The patient was informed of the diagnosis of cesarean scar pregnancy and the management options, including conservative and nonconservative treatments.

Finally, hysterectomy was performed, according to the patient's wishes. The total blood loss was 800 $\mathrm{mL}$, but no blood transfusion was required. The uterine cavity was filled with blood clots, and at the previous cesarean scar the myometrium was noted to be deficient owing to invasion of the chorion (Fig. 4). Histological examination revealed a lack of
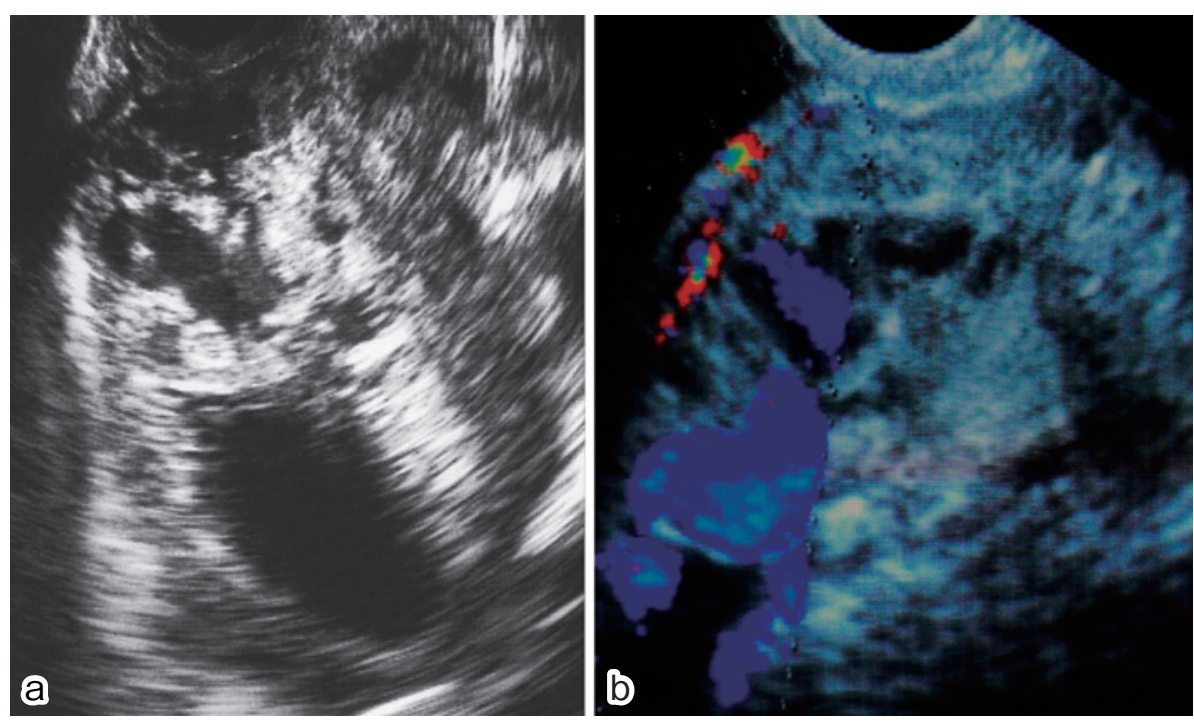

Fig. 2 Transvaginal ultrasonographic image at 9 weeks' gestation. a: A bulging mass with multiple irregularly shaped hypoechoic areas at the lower segment of anterior uterine myometrium, strikingly similar to lacunae. b: Color Doppler imaging demonstrating richly perfused, lacunae-like hypoechoic areas. 


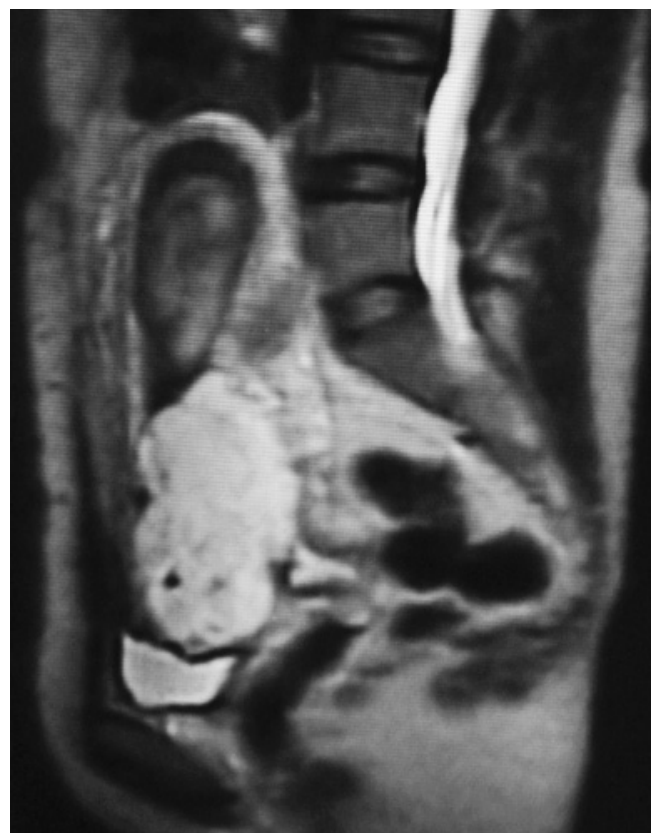

Fig. 3 Magnetic resonance image. Extreme thinning of the myometrium at the lower anterior uterine wall due to placental invasion was visualized.

decidual tissue and the presence of a cluster of trophoblast cells in the smooth muscle bundles. The patient was discharged from the hospital on the $8^{\text {th }}$ postoperative day without complications.

\section{Discussion}

If cesarean scar pregnancy is diagnosed at an early stage, multiple treatment options are available, uterine rupture and hemorrhage can be avoided, and fertility can be preserved ${ }^{5,6}$. Accurate diagnosis is also necessary to avoid dilatation and curettage, which is contraindicated because of the risk of uterine rupture and severe hemorrhage.

The criteria previously proposed have been applied for the diagnosis of cesarean scar pregnancy. Vial et $\mathrm{al}^{7}$ proposed the following criteria: (1) the trophoblast must be located mainly between the bladder and the anterior uterine wall, (2) no fetal parts must be visible in the uterine cavity, and (3) on a sagittal view of the uterus through the amniotic sac, discontinuity should be demonstrated in the anterior wall of the uterus. The criteria proposed by Godin et al. ${ }^{4}$ were (1) empty uterus and empty cervical canal, (2) development of the sac in the

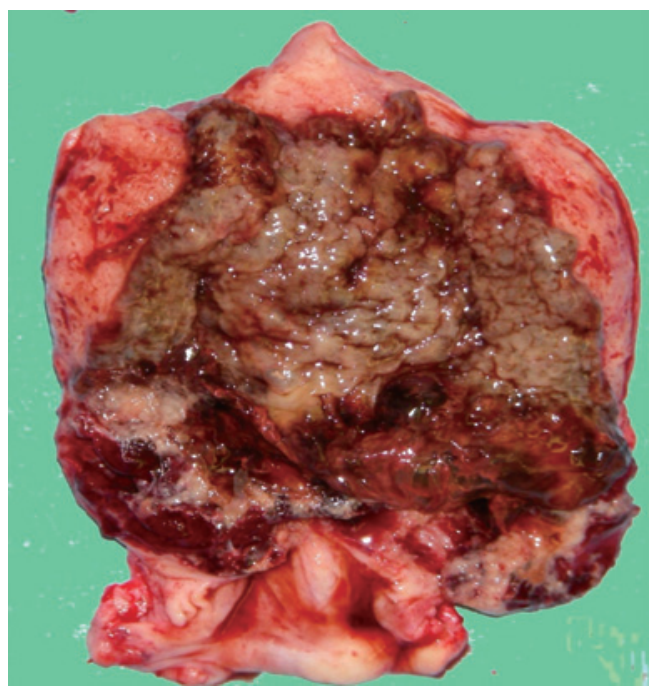

Fig. 4 Macroscopic view of the uterus. Enlarged uterine cavity with blood clots and a bulging mass in the lower segment of the anterior uterine wall.

anterior isthmic portion, and (3) absence of healthy myometrium between the bladder and the sac. However, our case met neither of these sets of criteria, as the uterine cavity was not empty, and the site of the sac could not be determined.

An anterior bulging tumor with lacunae-like hypoechoic areas was the first distinct abnormality to be recognized using gray-scale imaging in the present case but is not included in the diagnostic criteria of Vial et $\mathrm{al}^{7}$ or of Godin et $\mathrm{al}^{4}$. The ultrasonographic images bore no resemblance to typical ultrasonographic images in cesarean scar pregnancies which show an isolated gestational sac in the myometrium ${ }^{2-4}$. In previous reports of cesarean scar pregnancy, Einenkel et al. $^{5}$ and Kung et al. $^{6}$ described similar irregular masses in the uterine wall with serpentine and sonolucent areas. These images also resemble lacunae, which are generally considered to be characteristic of placenta accreta $^{8.9}$. The present case, together with these cases, suggests that the presence of lacunae-like hypoechoic areas should be also considered as one of the findings indicating cesarean scar pregnancy in the first trimester.

The resemblance of ultrasonographic images of cesarean scar pregnancy to those of placenta accreta raises the question of a similarity in pathology. The conditions usually appear in different periods of 
gestation and are classified as ectopic pregnancy and intrauterine pregnancy, respectively. However, both conditions are associated with risks of uterine rupture and massive hemorrhage due to direct invasion of trophoblast cells into the myometrum. Jurkovic et al. $^{2}$ have suggested that the term "abnormally adherent trophoblast/placenta" is more appropriate than "intramural pregnancy" to describe cesarean scar pregnancy. The similarity of ultrasonographic findings may be assumed to reflect the similarity of progression in these two conditions.

In summary, the present case demonstrates the importance for the diagnosis of cesarean scar pregnancy of the ultrasonographic finding of an anembryonic mass in the lower anterior uterine wall with lacunae-like hypoechoic areas. Such lacunae might not be restricted to placenta accreta and should also be noted as an expression of aggressive invasion of trophoblasts into the mural zone, even in the first trimester, long before the placenta is fully developed.

\section{References}

1. Rotas MA, Haberman S, Levgur M: Cesarean scar ectopic pregnancies: Etiology, diagnosis, and management. Obstet Gynecol 2006; 107: 1373-1381.

2. Jurkovic D, Hillaby K, Woelfer B, Lawrence A, Salim R, Elson CJ: First trimester diagnosis and management of pregnancies implanted into the lower uterine segment cesarean section scar. Ultrasound Obstet Gynecol 2003; 21: 220-227.

3. Seow KM, Huang LW, Lin YH, Lin MY, Tsai YL, Hwang JL: Cesarean scar pregnancy: issues in management. Ultrasound Obstet Gynecol 2004; 23: 247-253.

4. Godin PA, Bassil S, Donnez J: An ectopic pregnancy developing in a previous caesarean section scar. Fertil Steril 1997; 67: 398-400.

5. Einenkel J, Stumpp P, Koesling S, Horn LC, Hoeckel M: A misdiagnosed case of caesarean scar pregnancy. Arch Gynecol Obstet 2005; 271: 178-181.

6. Kung FT, Huang TL, Chen CW, Cheng YF: Cesarean scar ectopic pregnancy. Fertil Steril 2006; 85: 1508-1509.

7. Vial Y, Petignat P, Hohlfeld P: Pregnancy in a cesarean scar. Ultrasound Obstet Gynecol 2000; 16: 592-593.

8. Finberg HJ, Williams JW: Placenta accreta: Prospective sonographic diagnosis in patients with placenta previa and prior cesarean section. J Ultrasound Med 1992; 11: 333-343.

9. Comstock CH, Love JJ Jr, Bronsteen RA, et al:: Sonographic detection of placenta accreta in the second and third trimesters of pregnancy. Am J Obstet Gynecol 2004; 190: 1135-1140.

(Received, March 19, 2012)

(Accepted, April 26, 2012) 